International Journal of Pure and Applied Mathematics

Volume 96 No. 3 2014, 353-362

ISSN: 1311-8080 (printed version); ISSN: 1314-3395 (on-line version)

url: http://www.ijpam.eu

doi: http://dx.doi.org/10.12732/ijpam.v96i3.6

\title{
APPLICATION OF THE INFORMATION TECHNOLOGIES FOR SOLVING EXTREMAL PROBLEMS IN TEACHING MATHEMATICS
}

\author{
Vania Bizova-Laleva ${ }^{1} \S$ Kosta Gyrov $^{2}$ \\ 1,2 Plovdiv University \\ 236, Bul. Bulgaria, Plovdiv, BULGARIA
}

AMS Subject Classification: 97D40, 97U60

Key Words: teaching in mathematics, extremal problems, GeoGebra

\section{Introduction}

The topic "The least and greatest values of quadratic functions on a closed interval" is a required part of the 10th grade mathematics curriculum in Bulgarian High Schools [2]. The usual statement of the problem is: Find the least/greatest value of the function $f(x)=a x^{2}+b x+c$ on the interval $[p, q]$. In the particular problems only the type of the function and the interval are changeable.

This approach restricts us to solving only one type of problems. At the same time a whole bunch of problems are missing such as:

- describing adequately real world situation;

- applications of theoretical results for solving real world problems;

- illustrating how a real world problem could be solved;

- illustrating the application of modern technological tools for its solving.

Received: July 2, 2014

(C) 2014 Academic Publications, Ltd.

${ }^{\S}$ Correspondence author url: www.acadpubl.eu 
It is absolutely necessarily to be changed the approach for solving of extrema problems and to be shown deeply the connection between Mathematics and realworld life. It could be used to motivate students for deliberating utilization of this interesting and useful topic which is widely used in real life.

Some very good methods for solving extremal problems by application of technological capabilities of the programs Graf and MATLAB are given in [1] and [3]. The main disadvantage is these softwares are not taught in Bulgarian secondary school.

In this paper we present some practical problems solved in Math elective course in 10 Grade. The main goal of the paper is to set up and to describe the methodological approach for applications of mathematical model based on finding the extremal values of an analytically given quadratic function which domain is a closed interval on the real number line. A methodical technique for calculating these values by the technological capabilities of GeoGebra is described.

We will suggest the following algorithm for solving the given problem:

\section{A. Modeling}

A1. Analysis;

A2. Building of a mathematical model.

A3. Formulation of the mathematical problem.

B. Solving of the mathematical problem and obtaining the extremal values of the quadratic function on a closed interval by application of GeoGebra.

B1. Analysis and selection of methods and approaches for solving;

B2. Application of the approach chosen.

B2.1. Planning simulation: Select the parameter $m$ for simulation and determine: range of motion; step of movement; initial value $m_{0}$, and rate of change;

B2.2. Graph the quadratic function;

B2.3. Find the point $(m, f(m))$ on the graph of the function $f(x)$, where $m=m_{0}$;

B2.4. Simulation: Start the process of calculating and recording values of $f(x)$ for all values of the selected parameter $m$;

B2.5. Result: It is obtained the least and greatest function values for the corresponding values of the argument $x$. 
C. Adaptation of the obtained results to the practical task. (Interpretation, evaluation, etc.).

\section{Examples}

We will consider some particular problems which are appropriate for realizing the formulated above task by the suggested algorithm.

Practical problem 1. The base of the greenhouse has a shape of a square $11 \times 11 \mathrm{~m}$. Two square beds (see Figure 1 ) must be planted with two types of flowers. Find the dimensions of the beds with the least common area?

Solution: Implementation of the suggested above algorithm:

\section{A. Modeling}

A1. Analysis: The length of the bed's sides are natural numbers (in meters) and their sum is $11 \mathrm{~m}$. We have to obtain the lengths such that the sum of the areas of both beds be smallest.

A2. Building of a mathematical model: Denote the lengths of the side of the first bed and the second one by $x$ and $y$, correspondingly. Therefore, $x$ and $y$ are natural numbers: $1 \leq x \leq 11$ and $1 \leq y \leq 11$ and $x+y=11$, or $y=11-x$. Therefore, $x \in\{1,2,3, \ldots, 10\}$.

The sum of areas of the beds with lengths of sides $x$ and $11-x$ will be $f(x)=x^{2}+(11-x)^{2}=2 x^{2}-22 x+121$.

We have to obtain the lengths of the sides of the beds such that the sum of areas is minimal.

Comment: The modeling process consists of the following steps:

A2.1. Selecting a variable;

A2.2. Determine research space;

A2.3. Establishment of the quadratic function.

A3. Formulation of the mathematical problem. Find the values of $x \in$ $\{1,2,3, \ldots, 10\}$, for which the function $f(x)=2 x^{2}-22 x+121$ has a minimal value. 


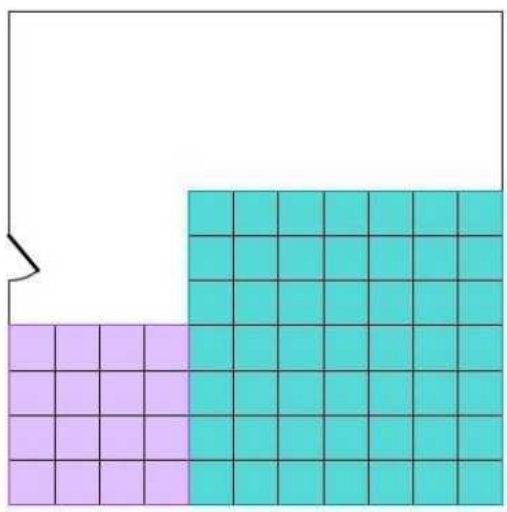

Figure 1.

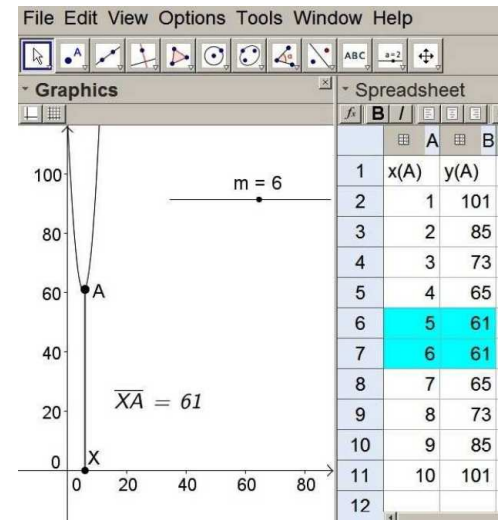

Figure 2.

B. Solving of the mathematical problem and obtaining the extremal values of the quadratic function on a closed interval by application of GeoGebra.

We will present two different scenarios for teaching the step B by application of interactive dynamic environment GeoGebra.

Scenario 1. Graphic method using a spreadsheet:

B1. Analysis and selection of methods and approaches for solving: Due to the type of the set $x \in\{1,2,3, \ldots, 10\}$ we write the values of the function $f(x)=2 x^{2}-22 x+121$ in spreadsheet and we select the smallest value of the function and the corresponding values of the argument $x$.

B2. Application of the approach chosen

B2.1. Planning simulation: Select a parameter $m$ for simulation and define

- Range of motion: It depends on the set on which the extrema of the function is looking for: the interval $[1,10]$.

- Step movement: It is 1 because $x \in\{1,2,3, \ldots, 10\}$.

- Initial value: Any value from the set $\{1,2,3, \ldots, 10\}$ could be an initial value. For example, let $m_{0}=1$.

- Rate of change: Its value has provide a sufficiently clear visualization of the process. Let it be 1 .

B2.2. Construct a graph of the quadratic function $f(x)=2 x^{2}-22 x+121$.

B2.3. Construct the point $\left(m_{0}, f\left(m_{0}\right)\right)$ on the graph.

B2.4. Simulation: Start the process of calculating and recording values of $f(x)$ for $m \in[1,10]$. 
B2.5. Result: We obtain the least value $f(x)=61$ and the corresponding values of the argument $x=5$ and $x=6$ (Figure 2).
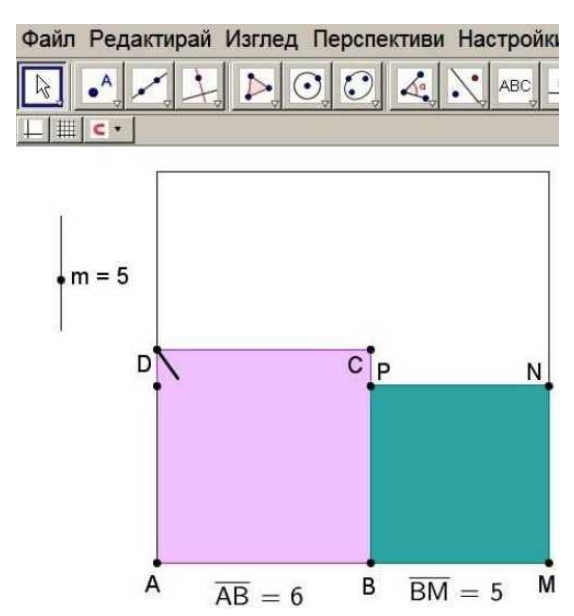

Figure 3 .
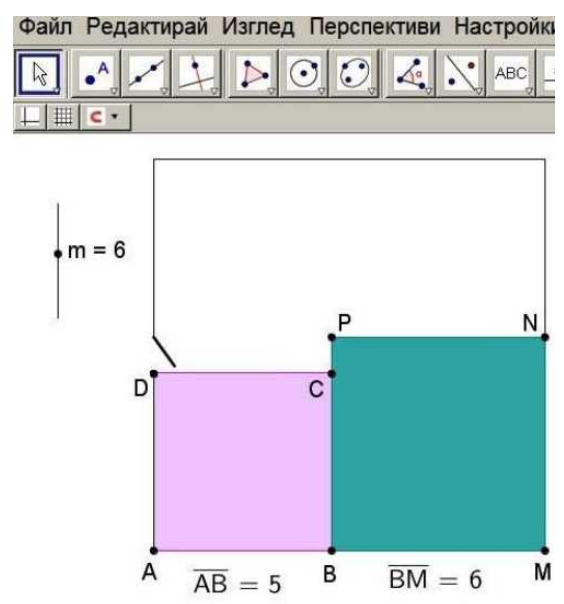

Figure 4.

C. Adaptation of the results to the practical task: There are two possible options for the size and location of the beds: $A B=6$ and $B M=5$ (Figure 3 ) or $A B=5$ and $B M=6$ (Figure 4). The second one (Figure 3 ) is not a solution of the practical task because the greenhouse' door can not be opened.

Scenario 2. Graphic method by visualization values of the function. Steps B1 and B2.1-B2.4 are the same as in the Scenario 1.

B2.5. Result: From the graph of the function we obtain the least value of $f(x)=61$ and the corresponding values of the argument $x=5$ and $x=6$ (Figure 5). 
File Edit View Options Tools V
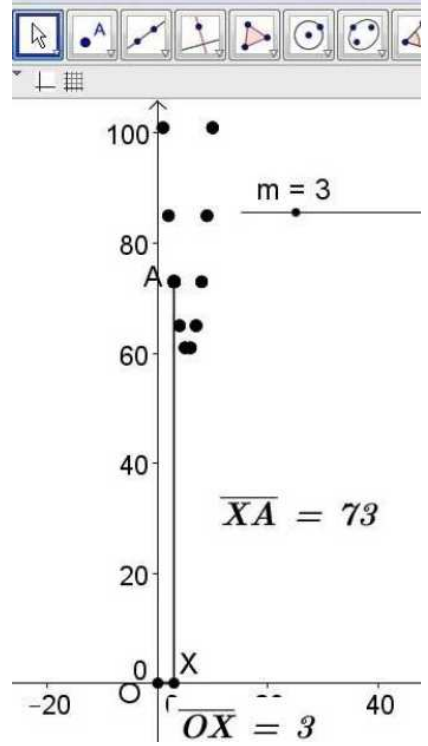

Figure 5.1.

File Edit View Options Tools W
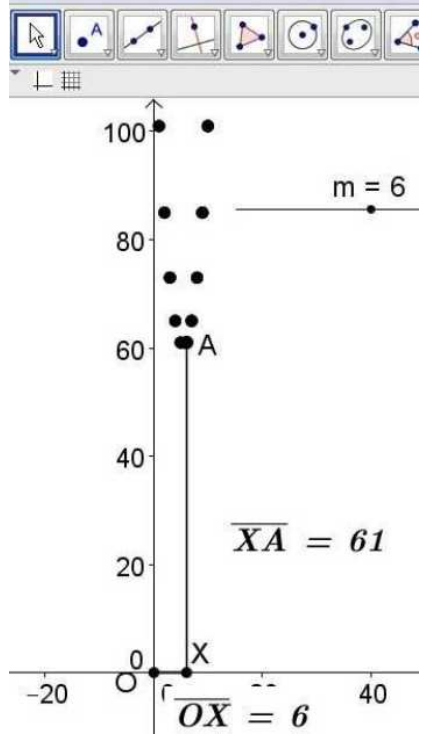

Figure 5.3.
File Edit View Options Tools V

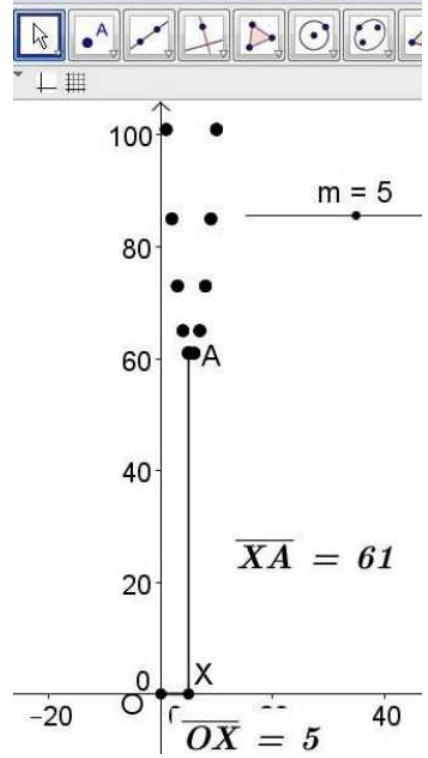

Figure 5.2.

File Edit View Options Tools Windo

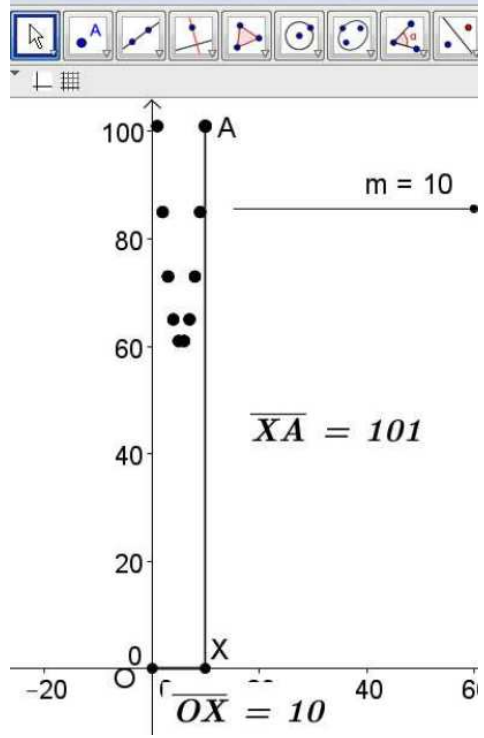

Figure 5.4.

Practical problem 2. The main dish of a restaurant is "specialty of the house". The cost for preparing a portion is 5.00 lev. When the selling price 
per portion is 10.00 lev the restaurant sells 3000 meals monthly. The manager knows the increase of the selling price by one lev lead to a decrease of the meal consumption by 300 units monthly and the profit from sales increases. He wants to know which selling price of the main dish will maximize the profit.

Solution: Implementation of the suggested above algorithm:

\section{A. Modeling}

A1. Analysis:The restaurant's profit depends on the profit of unit sales of meals and the number of sold meals.

A2. Building of a mathematical model: Denote the selling price of the "specialty of the house" by $x$, where $x$ is a positive real number $\left(x \in Q_{+}\right)$.

The profit $p$ of a meal is equal to the difference between the selling price and the costs, i.e. $p=x-5$, where $x \geq 5$.

The increase in the selling price is equal to the difference between the new price and the old one, i.e. $x-10$ (lev). Then the monthly sales will be reduced by $300(x-10)$. The number $n$ of monthly sold meals is equal to the difference between the initial sales and the reduced ones, i.e. $n=3000-300(x-10)=300(20-x)$ where $x \geq 20$.

The profit of the restaurant will be

$f(x)=p n=300(20-x)(x-5), x \in[5,10]$.

The values of $x$ will be calculated with an accuracy of 0.01 (the measurement of the price is up to 1 stotinka).

A3. Formulation of the mathematical problem: Find the values of $x \in$ $[5,10]$ for which the function $f(x)=300(20-x)(x-5)$ has the greatest value.

B. Solving of the mathematical problem and obtaining the extremal values of the quadratic function on a closed interval by application of GeoGebra

B1. Analysis and selection of methods and approaches for solving: Put values of the function $f(x)=300(20-x)(x-5)$ in spreadsheet for $x \in[5,10]$, select the greatest value of $f(x)$ and the corresponding values of the argument $x$.

B2. Application of the approach chosen. 


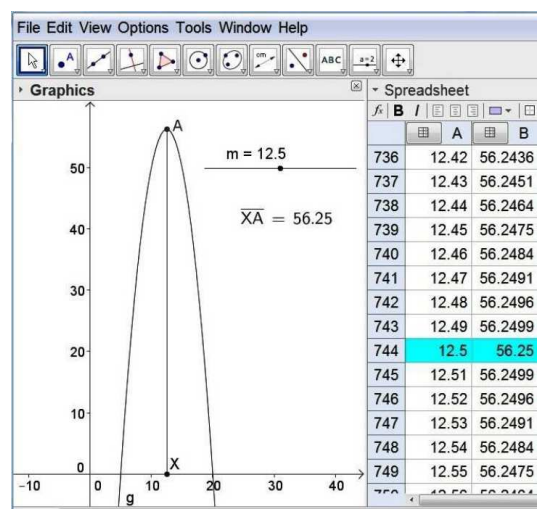

Figure 6.

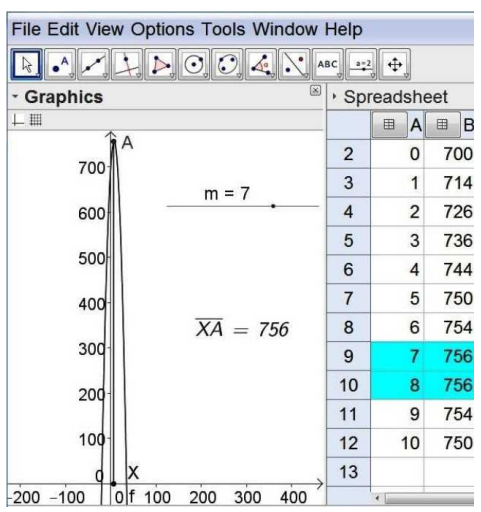

Figure 7.

B2.1. Planning simulation: Select a parameter $m$ for simulation and we define: the range of motion is $[5,10]$; the step movement is 0.01 ; the initial value is $m_{0}=5$; and the rate of change is 0.01 .

B2.2. Construct the graph of the function $g(x)=(20-x)(x-5)$.

B2.3. Construct a point $\left(m_{0}, g\left(m_{0}\right)\right)$ on the graph.

B2.4. Simulation: Start the process of calculating and recording values of $g(x)=(20-x)(x-5)$ for all values of $m \in[5,10]$.

B2.5. Result: From the spreadsheet we obtain the least value of $g(x)=$ 56.25 and its corresponding value of the argument $x=12.50$ (Figure 6).

The greatest value of the function $f(x)$ is $300 g(x)=16875$.

C. Interpretation of the obtained results to the practical task: The greatest monthly profit is $16875 \mathrm{lev}$ for selling unit price $12.50 \mathrm{lev}$.

Practical problem 3. A security company has a parking lot with 30 places. A lease with a logistics company is signed under the following conditions: the monthly unit rent is $35 \mathrm{lev}$ for up to 20 parking lots. For each additional parking lot over 20 ones there will be a discount of $1 \mathrm{lev}$ in the unit rent for each parking lot.

A) Which number of rented parking lots will guarantee the greatest profit of the security company?

B) Which number of leased parking lots will guarantee the greatest profit for the logistics company?

Solution: The implementation of the algorithm is similar to Problem 1 and 2 and we will emphasize only on some points. 


\section{A1. Analysis:}

A) The profit of the security company depends on the number of leased parking lots and the profit of each of them.

B) The contract is the most profitable for the logistics company if it leases the most parking places for the least possible amount.

A2. Building of a mathematical model: Denote by $x$ the number of the additional parking lots over $20, x \in\{1,2,3, \ldots, 10\}$. The total number of the leased parking lots is $n=20+x$ and the rent $p=35-x$. The income of the security company (rent of the logistics company) depends on the number of rented (leased) parking lots and the profit(rent) of one parking lot and it is $f(x)=p n=(35-x)(20+x)$.

A3. Formulation of the mathematical problem: Find the values of $x \in\{1,2,3$ $, \ldots, 10\}$ such that the function $f(x)$ has the greatest value.

B2. Application of the approach chosen: $m$ is the simulation parameter, the range of motion is $[0,10]$; the step movement is 1 ; the initial value is $m_{0}=0$; the rate of change is 1 .

B2.5. Result: From the spreadsheet we obtain:

A) the greatest value $f(x)=756$ for $x=7$ and $x=8$.

B) For the greatest value of the argument $x=10$ we have $f(10)=f(5)=$ $750<f_{\max }$ (Figure 7).

C. Interpretation of the obtained results to the practical task:

A) The greatest monthly profit of the security company is $756 \mathrm{lw}$, when 7 or 8 additional parking lots are rented by the logistics company, i.e. the total number of rented parking lots is 27 or 28 .

B) The logistic company has to rent additionally 10 parking lots, i.e. the total rented parking lots are 30 , and the rent will be $750 \mathrm{lw}$, which is the same as the rent for 25 parking lots.

\section{Summary}

By the above suggested innovative methodical method for solving extremal problems it could be reached: 
- better motivation for studying Mathematics;

- more communication knowledge of students;

- active involvement of students in the educational process;

- increasing the quality and effectiveness of the teaching of Mathematics.

\section{Acknowledgments}

The research was partially supported by the grant MU13FMI002, PU "Paisii Hilendarski".

\section{References}

[1] S. Grozdev, D. Dekov, Extremal problems in middle school by the help with computer tables, Mathematics and Informatics, I.VI., 4, (2013), 351-367.

[2] Dodunekov St., at all, Mathematics - specialized education in 10th Grade, Regalia 6, Sofia (2002).

[3] Karoleva St., Effective teaching on optimization problems by modeling and solving with MATLAB of practical problems, In: Proc. Of 40th Anniversary Spring Conference of the Union of Mathematicians in Bulgaria, Borovec, 5-9 April, 2011, 406-412.

[4] http. www.geogebra.org (last visited on 10.07.2014) 